EPiC Series in Engineering
Volume 3, 2018, Pages 217-226
HIC 2018. 13th International
Conference on Hydroinformatics

\title{
Topographic analysis of wetlandscapes: fractal dimension and scaling properties
}

\author{
L. E. Bertassello ${ }^{1 *}$, P. S. C. Rao ${ }^{1,2}$, G. Botter ${ }^{3}$ and A. F. Aubeneau ${ }^{1}$ \\ ${ }^{1}$ Lyles School of Civil Engineering, Purdue University, West Lafayette, IN $47907-$ \\ 2051, USA \\ ${ }^{2}$ Agronomy Department, Purdue University, West Lafayette, IN 47907-2054, USA \\ ${ }^{3}$ Department of Civil, Architectural and Environmental Engineering, University of \\ Padua, I-35100 Padua, Italy
}

Corresponding author: Leonardo Enrico Bertassello(lbertass@purdue.edu)

\begin{abstract}
Wetlands are ubiquitous topographic depressions on landscapes and form critical elements of the mosaic of aquatic habitats. The role of wetlands in the global hydrological and biogeochemical cycles is intimately tied to their geometric characteristics. We used DEM analysis and local search algorithms to identify wetland attributes (maximum stage, surface area and storage volume) in four wetlandscapes across the United States. We then derived the exceedance cumulative density functions (cdfs) of these attributes for the identified wetlands, applied the concept of fractal dimension to investigate the variability in wetland' shapes. Exponentially tempered Pareto distributions were fitted to DEM derived wetland attributes. In particular, the scaling exponents appear to remain constant through the progressive water-filling of the landscapes, suggesting self-similarity of wetland geometrical attributes. This tendency is also reproduced by the fractal dimension (D) of wetland shorelines, which remains constant across different water-filling levels. In addition, the variability in D is constrained within a narrow range $(1<\mathrm{D}<1.33)$ in all the four wetlandscapes. Finally, the comparison between wetlands identified by the DEM-based model are consistent with actual data.
\end{abstract}




\section{Introduction}

Wetlands are critical elements of landscape aquatic habitat mosaics. Some of their functions impact the physical and biogeochemical process dynamics, which, in turn, influence the ecological integrity of terrestrial and aquatic habitats (Cohen, et al., 2016; Rains, et al., 2016). Natural landscapes characterized by multiple wetlands (hereafter wetlandscapes) occur as a result of complex interactions between climate, terrain, soil, water availability and biota (Whittaker, 1975). Formal analyses of topographic data suggest that some landscapes may be either self-similar or self-affine (Gallant, Moore, Hutchinson, \& Gessler, 1994; Mandelbrot, 1983; Turcotte, 1997; Rodriguez-Iturbe \& Rinaldo, 2001). Intersecting a horizontal plane through a self-affine fractal surface (e.g., topographic surface), the points where fractal surface returns to the horizontal plane form a fractal known as random Cantor set (Russ, 1994; Seekell, Pace, Tranvik, \& Verpoorter, 2013). The direct analogy of this approach is to measure the areas of the wetlands (or lakes) which lie below this threshold. In particular, the intersection of the horizontal plane with the self-affine fractal surface produces boundary line which are self-similar rather than merely self-affine. Therefore, if we repeat the process for a fractal surface using several thresholds, we obtain several sets of self-similar wetlandscapes characterized by constant values for wetland shorelines fractal dimension $(D)$. In particular, D constrained between $D=1$ (a population of perfectly circular and smooth shorelines) and $D=2$ (a population of shorelines so irregular they are space filling). However, especially in steep slope conditions, slicing the landscape using a horizontal plane may underestimate the number and the size of the wetlands. For this reason, we applied the topographic depression identification (TDI) model (Le \& Kumar, 2014), to delimitate depressions in the landscape; this procedure identifies all depressions that might be wetlands (i.e. potential wetlands) only they meet various criteria for being so classified. The TDI model was run for several levels of wetland filling to simulate the slicing of the landscape at different threshold levels. Then we characterized the exceedance cumulative density functions, cdfs, of wetland attributes such as maximum stage, surface area and storage volume and, the wetland shoreline fractal dimension to scrutinize the complexity and variability of their shapes. Finally, to validate our approach, we compare the size distribution of all wetlands identified by the model with the wetlands listed in the U.S. National Wetland Inventory (NWI) for the four regions we investigated.

\section{Material and Methods}

\subsection{Identification of wetlands from DEMs}

In this study, we follow the recent framework developed by Chu et al (2010), Shaw et al (2012), Chu et al (2013), and improved by Le and Kumar (2014). Firstly, the center of each wetland is determined by applying the D8 algorithm proposed by O'Callaghan and Mark (1984). This method calculates flow direction for the processed cell by examining the elevation of its eight neighbors and determining drainage direction as the neighboring cell with the steepest elevation descent. If the cell has no neighbors with lower elevation, it is identified as the lowest elevation points (local minima in the landscape) of the wetland. Starting from the center of each potential wetland, local search algorithm is then implemented to identify the depression cells (Chu, Chi, Zhang, \& Yang, 2010). Such algorithm ends once that a threshold is identified. This threshold represents the highest elevation for water ponding, beyond which water spills. At the threshold level, a wetland reaches its maximum value for stage, area and storage volume. The elevation of the wetland threshold (local maxima in the landscape) controls the shift from wetland-filling to wetland-spilling and/or merging. Hydro-climatic forcing, such as rainfall and evapotranspiration, may alter the water level inside wetlands, represented 
as the changing threshold limit. An intense rainfall event, or a prolonged flooding period, results in wetland-merging once the water level reaches their common threshold. To simulate the filling of each wetland, the DEM is then edited by raising the depression cell to its threshold level. Then, the flow direction and the local searching algorithm are implemented again to derive the maximum values for stage, area and volume under this new condition.

Following Chu et al (2010) and Le and Kumar (2014), the geometric attributes of the wetlands in terms of stage, area and volume are estimated from the output of the previously defined model. The surface area of any wetland represents the maximum flooded surface area that can be observed in the wetland at the threshold elevation. Therefore, the surface area, $A_{k}^{(l)}\left[\mathrm{L}^{2}\right]$, of the k-th depression at that level, $l$, is the sum of the areas of all individual cells within that depression. The perimeter is derived by calculating the distance between each adjoining pair of pixel around the border of the region. The stage value inside each wetland can be determined by evaluating the difference in elevation between the threshold and the lowest grid cell that compose the wetland. This difference represents the maximum depth of the wetland for that specific level:

$h_{k, \max }^{(l)}=\max _{1 \leq i \leq m_{k}}\left(z_{k, t}^{(l)}-z_{k, i}^{(l)}\right)$

where $m_{k}$ is the number of cells $z_{k, t}^{(l)}$, [L] is the elevation of the threshold level and $z_{k, i}^{(l)}[\mathrm{L}]$ is the elevation of the ith cell, respectively in the kth wetland at level 1 . The storage volume, $\mathrm{V}$, of any wetland can be calculated by summing the contribution of each grid cell. The storage volume of the kth wetland at level 1 , denoted as $V_{k}^{(l)}\left[\mathrm{L}^{3}\right]$, is given by:

$V_{k}^{(l)}=\sum_{i=1}^{m_{k}}\left(z_{k, t}^{(l)}-z_{k, i}^{(l)}\right) \Delta x \Delta y$

where, $\Delta x[\mathrm{~L}]$ and $\Delta y[\mathrm{~L}]$ are the uniform horizontal resolution of the DEM data.

\subsection{Fractal dimension of the identified wetlands}

Fractal dimension has been used to describe the geometry of irregular objects for decades (Mandelbrot, 1983; Krummel, Gardner, Sugihara, O'Neill, \& Coleman, 1987; Lovejoy, 1982; Sugihara \& May, 1990)[13]. Lovejoy (1982) used the perimeter-area method proposed by Mandelbrot (1983) to calculate the fractal dimension of natural planar objects. The perimeter-area method quantifies the degree of complexity of planar shapes. The degree of complexity of a polygon is characterized by the fractal dimension, $D$, such that the perimeter, $P$, of a patch (wetland) is related to its area, $A$, by $P \approx A^{D / 2}$. For circles, $P \approx \sqrt{A}$ and $D=1$. As the polygons become more complex, the perimeter becomes increasingly dissected (plane filling) or elongated (e.g., riverine wetlands) and $P \approx$ $A$ with $D=2$. In particular, fractal dimension, $D$, of the shorelines of wetlands can be derived from the slope of the regression line of a $\log -\log$ plot of $\mathrm{P}$ vs A. This slope is equal to half of the boundary fractal dimension (Russ, 1994).

\subsection{Geospatial data}

This framework has been applied to four wetlandscapes across the United States. Despite the topographic and hydro-climatic differences that characterized these landscape, the massive presence 
of wetlands directed us to choose these regions where apply our framework. The DEM data were downloaded from the United States Geological Survey (USGS) platform National Map Viewer (https://viewer.nationalmap.gov). The DEM data for the six locations are characterized by the same dimension $(10 \mathrm{~km} \times 10 \mathrm{~km})$ and the same resolution $(1 / 3$ arc-second). We compared the results obtained using our framework with data downloaded wetland maps for the same four regions from the U.S. National Wetlands Inventory (https://www.fws.gov/wetlands/). In particular, these wetland landscapes include: California vernal pools, North Dakota prairie potholes, Florida cypress domes and Texas playa lakes.

\section{Results and Discussion}

\subsection{Cumulative density functions of wetland stage, area and volume}

The outputs of the TDI model were used to evaluate the exceedance cumulative density functions $(c d f \mathrm{~s})$ of wetland attributes in terms of maximum stage, surface area and storage volume for different filling levels (Figure 1). With increasing filling level, the water level rises and covers larger portion of wetlands in the wetlandscape. In addition, the presence of larger wetlands can be induced by the merging of small wetlands that coalesced as the filling level increases. As the filling level is increased, the tails of wetland attribute cdfs extend because the larger wetlands are now filled. However, the cdfs retain the same shape. Analytical solutions for wetland size distribution have been the focus of several recent studies (Zhang, Schwartz, \& Liu, 2009; Shook, Pomeroy, Spence, \& Boychuk, 2013; Van Meter \& Basu, 2015). Generally, Pareto distribution $\left(P(X>x) \propto x^{-b}\right)$ is found to be appropriate for lake size distribution (Hamilton, Melack, Goodchild, \& Lewis, 1992) (Downing, et al., 2006), but many datasets show a tempering in the upper tail of such cdfs. Several reasons contribute to this tempering. For example, datasets over a limited area will not contain wetland large enough to extend the power law behavior that characterize the distribution of the smaller wetlands. Furthermore, some of the large waterbodies may be lost due to human alterations of the landscape that dried portions of the landscape. For this reason, we fitted the cdfs of surface area with an exponentially tempered Pareto distribution $\left(P(X>x) \propto x^{-b} e^{-c x}\right)$. In addition, the same analytical distribution is used for the fitting of the maximum stage and storage volume cdfs. The fitted cdfs are represented by the solid lines in Figure 1, while the parameters of the distribution are reported in Table 1.

\begin{tabular}{ccccccc}
\hline & \multicolumn{2}{c}{ Maximum Depth } & \multicolumn{2}{c}{ Surface Area } & \multicolumn{2}{c}{ Storage Volume } \\
\hline & $\mathrm{b}$ & $\mathrm{c}$ & $\mathrm{b}$ & $\mathrm{c}$ & $\mathrm{b}$ & $\mathrm{c}$ \\
\hline Califor & & $4.28 \cdot 10^{-1}$ & & $9.23 \cdot 10^{-5}$ & & $9.06 \cdot 10^{-5}$ \\
nia & $0.76 \pm 0.06$ & $471 \cdot 10^{-1}$ & $0.50 \pm 0.08$ & $5.73 \cdot 10^{-4}$ & $0.45 \pm 0.06$ & $2.79 \cdot 10^{-4}$ \\
& & $0.38 \cdot 10^{1}$ & & $2.72 \cdot 10^{-4}$ & & $2.20 \cdot 10^{-4}$ \\
Florida & $1.85 \pm 0.08$ & $0.50 \cdot 10^{1}$ & $0.26 \pm 0.12$ & $2.01 \cdot 10^{-3}$ & $0.95 \pm 0.12$ & $5.75 \cdot 10^{-4}$ \\
N. & & $4.01 \cdot 10^{1}$ & & $2.00 \cdot 10^{-6}$ & & $1.51 \cdot 10^{-5}$ \\
Dakota & $0.45 \pm 0.17$ & $4.10 \cdot 10^{1}$ & $0.88 \pm 0.16$ & $7.62 \cdot 10^{-6}$ & $0.49 \pm 0.07$ & $1.23 \cdot 10^{-3}$ \\
& & $2.56 \cdot 10^{-1}$ & & $7.39 \cdot 10^{-6}$ & & $2.42 \cdot 10^{-6}$ \\
Texas & $0.25 \pm 0.23$ & $3.58 \cdot 10^{-1}$ & $0.01 \pm 0.03$ & $1.13 \cdot 10^{-5}$ & $0.11 \pm 0.05$ & $1.56 \cdot 10^{-5}$ \\
\hline
\end{tabular}


Table 1: Parameters of the Tempered Pareto distribution for the modeled wetland maximum depth, surface area and storage volume in the four wetlandscapes. The mean of the scaling exponent, $b$, for the four level of filling is reported along with its standard deviation. The tempering parameter, $c$, is reported in terms of minimum and maximum value.

Tempered Pareto distribution fits well to the DEM-modelled data $\left(R^{2}>0.95\right)$ for all three attributes, with small tempering constant, $\mathrm{c}$, for maximum depth and storage volume distributions. The scaling coefficient, $b$, tends to be constrained around the mean value $(\sigma<0.1)$, while the tempering parameter, $c$, shows a decrease from level 1 to level 4 (Figure 1). This confirms that for higher level of filling, the probability of finding steeper and larger wetland increases. For wetland size distribution, the tempered Pareto reproduces the lower tail of the cdfs that describes the distribution of smaller wetlands; however, overall it underestimates the upper tail of the cdfs. The complexity of finding a proper analytical distribution that well reproduce the data is encountered in several studies (Shook, Pomeroy, Spence, \& Boychuk, 2013; Seekell \& Pace, Does the Pareto distribution adequately describe the size-distribution of lakes?, 2011). More heavy-tailed or hybrid distributions might be a solution for a proper fitting of the right side of such cdfs. If the goal is to well describe the more abundant smaller wetlands (ecologically important) the emphasis is on functions (e.g., log-normal) that best fit the smaller areas, while heavier tailed distributions (e., Pareto) might be preferred if the emphasis is on larger wetlands. Use of hybrid functions might also be another strategy. In spite of such limitations, tempered Pareto distributions fitted to our results suggest that wetlandscapes may exhibit self-similarity because the scaling properties (b) are maintained throughout the different levels of filling that are used to threshold the landscapes (Russ, 1994); the only difference among these distributions is expressed by the tempering parameter (c), which decreases for progressive filling levels.

The emergent self-similarity of wetlandscapes is also investigated though the analysis of wetland fractal dimension. Fractal dimension of wetlands shorelines is evaluated for the four landscapes using the perimeter and area obtained from TDI model. The left panels of Figure 2 compare the values of fractal dimension at different filling levels with the prescribed limit for circular wetland (black dashed line, $\mathrm{D}=1$ ). Except for Texas (Figure $2 \mathrm{D} 1$ ), which is mainly characterized by circular wetlands, in the other landscapes D tends to diverge from this boundary. However, the deviation from this lower limit appears to be constrained by an upper limit around $\mathrm{D}=1.33$ (Figures $2 \mathrm{~A} 2-\mathrm{D} 2$ ). This outcome is consistent with the analysis on Swedish lakes' fractal dimension by Cael \& Seekell (2016). This upper limit is theoretically prescribed by percolation theory, where the fractal dimension of the boundaries, which envelop a percolation cluster (e.g., wetland or lake), is predicted to be $D=4 / 3$.

Another key finding showed in Figure 2 is that, despite the increase in the threshold level, and therefore the degree of filling inside wetlands, their shape, and, therefore, their fractal dimension, $D$, is maintained. This represents a further hint that wetlandscapes are characterized by self-similarity. A self-similar fractal means that each piece of the object is geometrically like the whole, and the object can be enlarged or reduced without the scale being apparent (Rodriguez-Iturbe \& Rinaldo, 2001). In this case, wetlands can be enlarged or reduced by varying the filling level in the landscape. The shape of wetland shorelines and the concept of fractal dimension is a good tool from an eco-hydrological point of view. Variation in wetland attributes (e.g., perimeter-area ratio) is of key importance from an ecological perspective since species are strongly associated with wetland edges during breeding and non-breeding seasons (Werner, Skelly, Relyea, \& Yurewicz, 2007). Foraging habits of some marsh species indicate that edges between vegetation and open water are preferred for finding prey (Tozer, Abraham, \& Nol, 2006). 


\subsection{Comparison between wetlands identified by the TDI model and NWI dataset}

The local search algorithm that we applied in the current analysis was used mainly to derive small puddles (Chu, Yang, Chi, \& Zhang, 2013) and microtopographic depressions (Le \& Kumar, 2014) so far. To validate this approach also in larger depression such as wetlands, we compare the results obtained from this algorithm with actual wetland data downloaded from the NWI dataset (Figure 3).

The TDI algorithm was run for several times to reach convergence with the NWI dataset. In some cases, such as North Dakota and Texas it quickly reaches the convergence, while in the other landscapes (Florida and California) the algorithm was implemented for several levels. The main reason for this discrepancy is related to the type of wetlands that characterizes the landscapes. These landscapes are mainly composed by geographically isolated wetlands that are well dispersed; therefore, the local search is faster. Florida landscape is also mainly characterized by GIWs, but in this case Florida cypress domes are close to each other, leading to merging that quickly alters number and size of wetlands. For these reasons, it is necessary to fill the landscape by enough amount of water to resemble the data provided by the NWI. On the other hand, when wetlands are integrated in the river network or the landscape is influenced by human-induced activities, such as in California, the model algorithm is not performing well. The NWI database we use to evaluate TDI approach has well documented limitations (Tiner, 1997), with omission errors seriously underrepresenting small wetland prevalence and extent.

This is evident in Figure 3 where TDI algorithm finds several small potential wetlands that are not considered by the NWI dataset. The correct delineation of wetlands requires the knowledge of several criteria such as hydrophitic vegetation, hydric soil and wetland hydrology. The method that we used only considers topographic properties of the landscape since wetlands usually occupy areas at lowest elevation. The local search model we adopted in the current research is useful to estimate the small wetlands that are difficult to delimit and also could confirm the data provided by the NWI replacing some field measurement that are always difficult and require considerable effort. In addition, the TDI model could be applied in all the regions where wetland geometric data are not present in order to have a quick estimate about the presence of wetlands, since only DEM map are necessary.

\section{Conclusions}

The application of topographic search algorithm and fractal analysis represents a powerful tool to investigate the distribution and the geometrical properties of wetlandscapes. One of the main insight of the current analysis is represented by the emergent self-similarity that characterizes wetlandscapes. In particular, as the landscapes is progressively filled by water the scaling (b) of wetlands attributes (maximum stage, surface area and storage volume) distribution is maintained. This result is also suggested by the analysis of wetlands shorelines fractal dimension that appears to be constrained in a narrow range $(1<D<1.33)$, and whose pdfs is the same over different filling levels. Wetland attributes in terms of maximum stage, surface area and storage volume are fundamental both from a hydrological and ecological perspective, but real measurements of these quantities are often limited to few wetlands in a landscape. For this reason, DEM-based approaches are useful in order to give a first assessment about the distribution and location of wetlands, especially in those regions where wetland geometric data are not available. The TDI model provides data for stage, area and volume that characterize wetlands in landscapes. However, main limitation is in definition of wetland, considered simply as a portion of the landscape where water can accumulate. Therefore, integrations with hydrological analysis, vegetation distribution and LULC (land use land cover) changes are necessary to better delineate wetland attributes. 


\section{References}

Cael, B. B., \& Seekell, D. A. (2016). The size-distribution of Earth's lakes. Sci. Rep.

Chu, X., Chi, Y., Zhang, J., \& Yang, J. (2010). An improved method for watershed delineation and computation of surface depression storage. Innovations in Watershed Management Under Land Use and Climate Change, 1113-1122.

Chu, X., Yang, J., Chi, Y., \& Zhang, J. (2013). Dynamic puddle delineation and modeling of puddleto-puddle filling-spilling-mergin-splitting overland processes. Water Resour. Res.(49), 38253829.

Cohen, M. J., Creed, I. F., Alexander, L., Basu, N. B., Calhoun, A. J., Craft, C., . . Musg. (2016). Do geographically isolated wetlands influence landscape functions? Proceedings of the National Academy of Sciences(113), 1978-1986.

Downing, J. A., Prairie, Y. T., Cole, J. J., Duarte, C. M., Tranvik, L. J., Striegl, R. G., . . Middelburg, J. J. (2006). The global abundance and size distribution of lakes, ponds, and impoundments. Limnol. Oceanogr.(51), 2388-2397.

Gallant, J. C., Moore, I. D., Hutchinson, M. F., \& Gessler, P. (1994). Estimating fractal dimension of profiles: A comparison of methods. Math. Geol., 26(4), 455-481.

Hamilton, S. K., Melack, J. M., Goodchild, M. F., \& Lewis, W. M. (1992). Estimation of the fractal dimension of terrain from lake size distributions. Lowland Floodplain Rivers: Geomorphological Perspectives.

Krummel, J., Gardner, R., Sugihara, G., O'Neill, R., \& Coleman, P. (1987). Landscape Patterns in a Disturbed Environment. Oikos, 48(3), 321-324.

Le, P. V., \& Kumar, P. (2014). Power las scaling of topographic depressions and their hydrologic connectivity. Geophys. Res. Let.(41), 1553-1559.

Lovejoy, S. (1982). Area-perimeter relation for rain and clouds areas. Science(216), 185-187.

Mandelbrot, B. B. (1983). The fractal geometry of nature. New York: Freeman.

O'Callaghan, J. F., \& Mark, D. M. (1984). The extraction of drainage networks from digital elevation data. Comput. Vision Graphics Image Process., 28(3), 323-344.

Rains, M. C., Leibowitz, S. G., Cohen, M. J., Creed, I. F., Golden, H. E., Jawitz, J. W., . . . McLaughlin, D. L. (2016). Geographically isolated wetlands are part of the hydrological landscape. Hydrological Process(30), 153-160.

Rodriguez-Iturbe, I., \& Rinaldo, A. (2001). Fractal River Basins: Chance and Self-Organization. New York: Cambridge University Press.

Russ, J. C. (1994). Fractal Surfaces. New York: Plenum.

Seekell, D. A., \& Pace, M. L. (2011). Does the Pareto distribution adequately describe the sizedistribution of lakes? Limnol. Oceanogr.(56), 350-356.

Seekell, D. A., Pace, M. L., Tranvik, L. J., \& Verpoorter, C. (2013). A fractal-based approach to lake size-distributions. Geophys. Res. Let.(40), 517-521.

Shaw, D. A., Pietroniro, A., \& Martz, L. (2012). Topographic analysis for the prairie pothole region of Western Canada. Hydrol. Process.

Shook, K., Pomeroy, J. W., Spence, C., \& Boychuk, L. (2013). Storage dynamics simulations in prairie wetland hydrology models: evaluation and parameterization. Hydrol. Process(27), 1875-1889.

Sugihara, G., \& May, R. M. (1990). Applications of fractals in ecology. Trends in Ecology \& Evolution, 79-86.

Tiner, R. J. (1997). NWI Maps: What They Tell Us. National Wetlands Newsletter, 7-12.

Tozer, D. C., Abraham, K. F., \& Nol, E. (2006). Improving the accuracy of counts of wetland breeding birds at the point scale. Wetlands, 518-527.

Turcotte, D. L. (1997). Fractal and chaos in geology and geophysics. Cambridge (U.K.): Cambridge University Press. 
Van Meter, K. J., \& Basu, N. B. (2015). Signatures of human impact: size distributions and spatial organization of wetlands in the Prairie Pothole landscape. Ecological Applications(25), 451465.

Werner, E. E., Skelly, D. K., Relyea, R. A., \& Yurewicz, K. L. (2007). Amphibian species richness across environmental gradients. $\operatorname{Oikos}(116), 1697-1712$.

Whittaker, R. H. (1975). Communities and Ecosystems. New York: Mac-Milan.

Zhang, B., Schwartz, F. W., \& Liu, G. (2009). Systematics in the size structure of prairie pothole lakes through drought and deluge. Water Resour. Res.
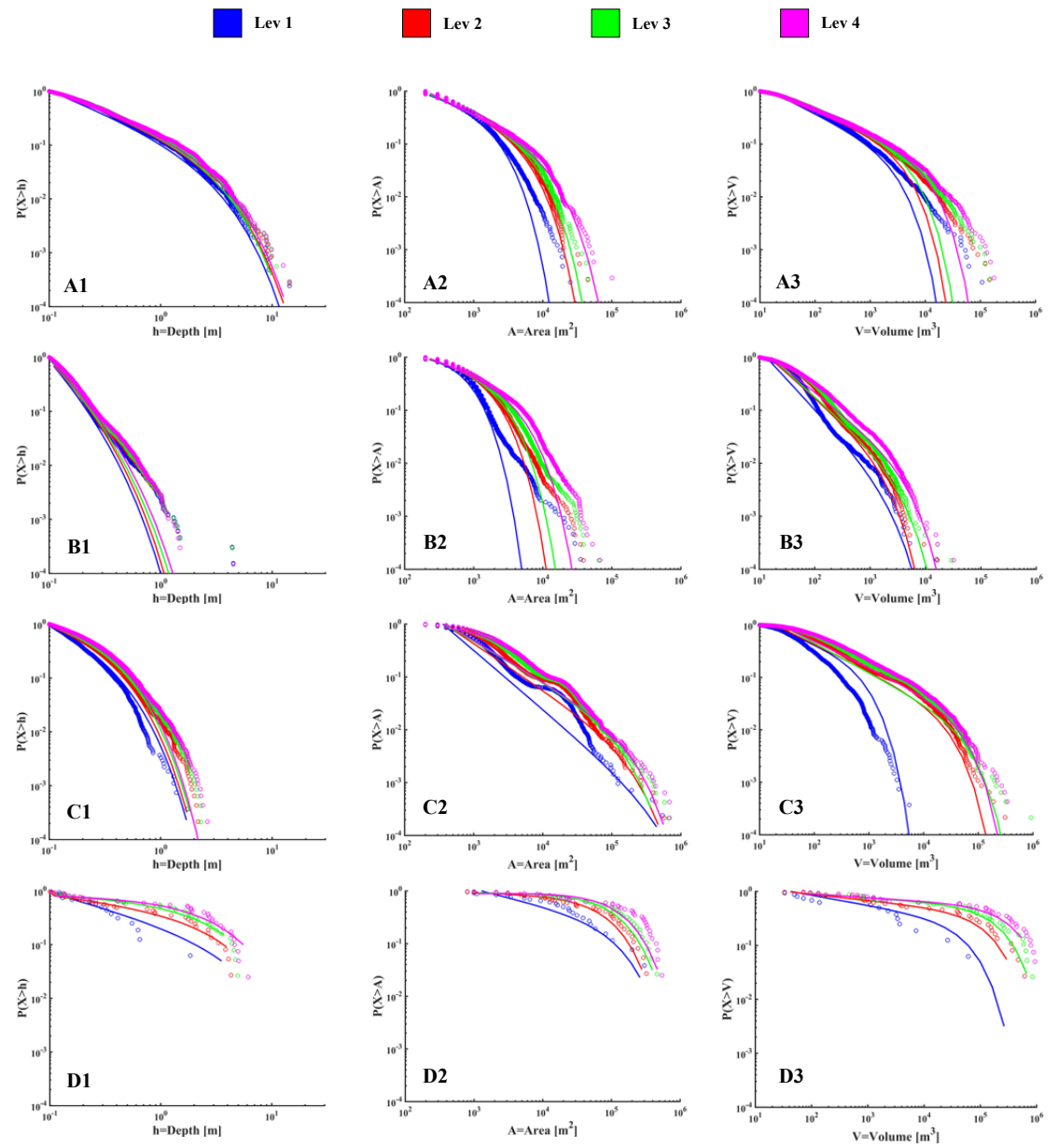

Figure 1: Comparison between the empirical exceedance cumulative density functions (circles) for wetland maximum depth (left column), surface area (center column) and storage volume (right column) obtained from modelled data and the tempered Pareto analytical distribution (solid lines) in the four wetlandscapes. The different colors used in the pictures stand for different level of filling. A1, A2, A3 report the cdfs in California, B1, B2, B3 in Florida, C1, C2, C3 in North Dakota and D1, D2, D3 in Texas. 

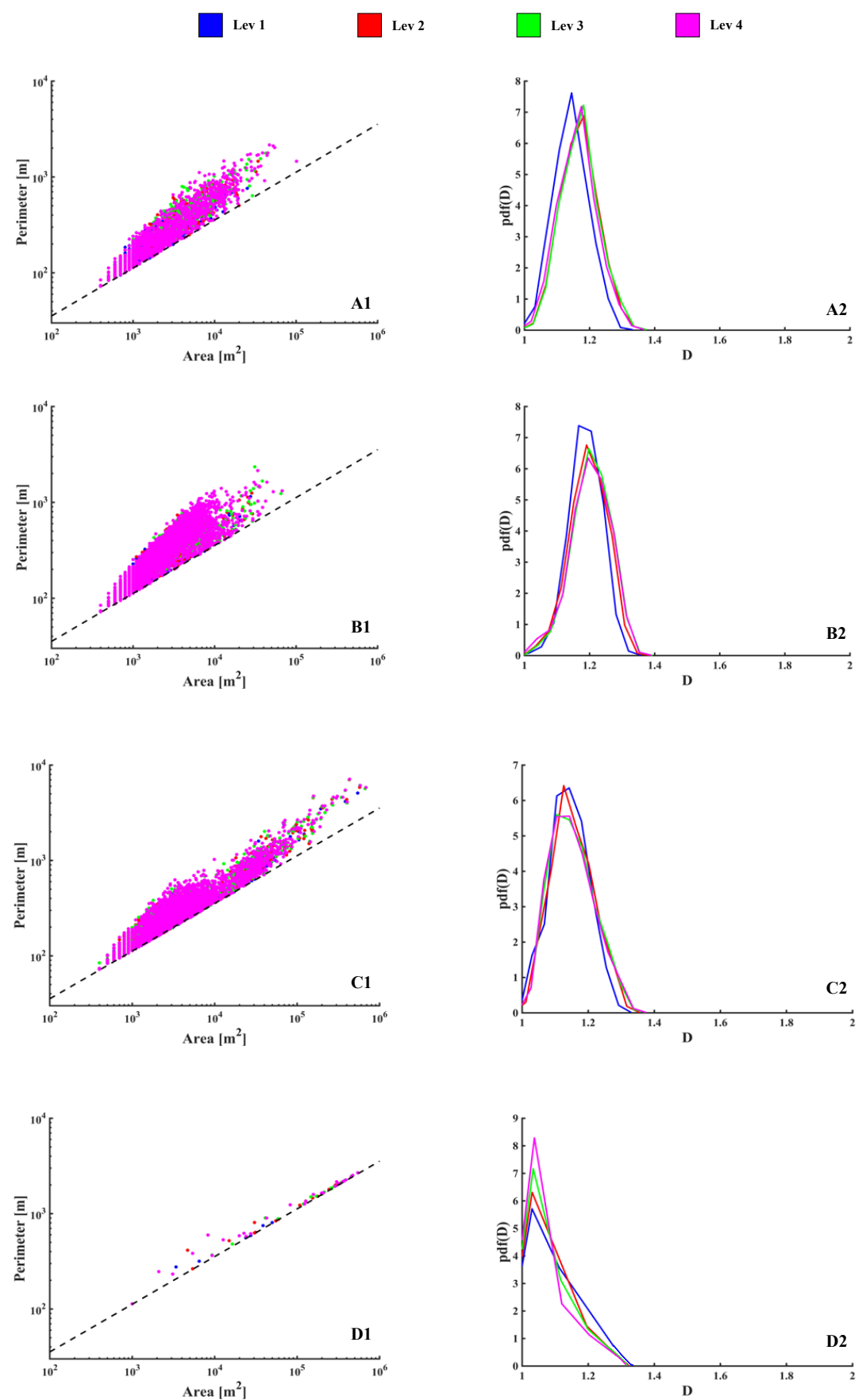

Figure 2: A1, B1, C1, D1 report the shoreline fractal dimension D for the four wetlandscapes data obtained from the TDI model. The black dashed line represents the lower limit for circular wetlands $(\mathrm{D}=1) . \mathrm{A} 2, \mathrm{~B} 2, \mathrm{C} 2, \mathrm{D} 2$ show the probability density function of the fractal dimension obtained from the perimeter to area ratio for the four filling level. A1, A2 report the data for California, B1, B2 for Florida, C1, C2 for North Dakota and D1, D2 for Texas. 

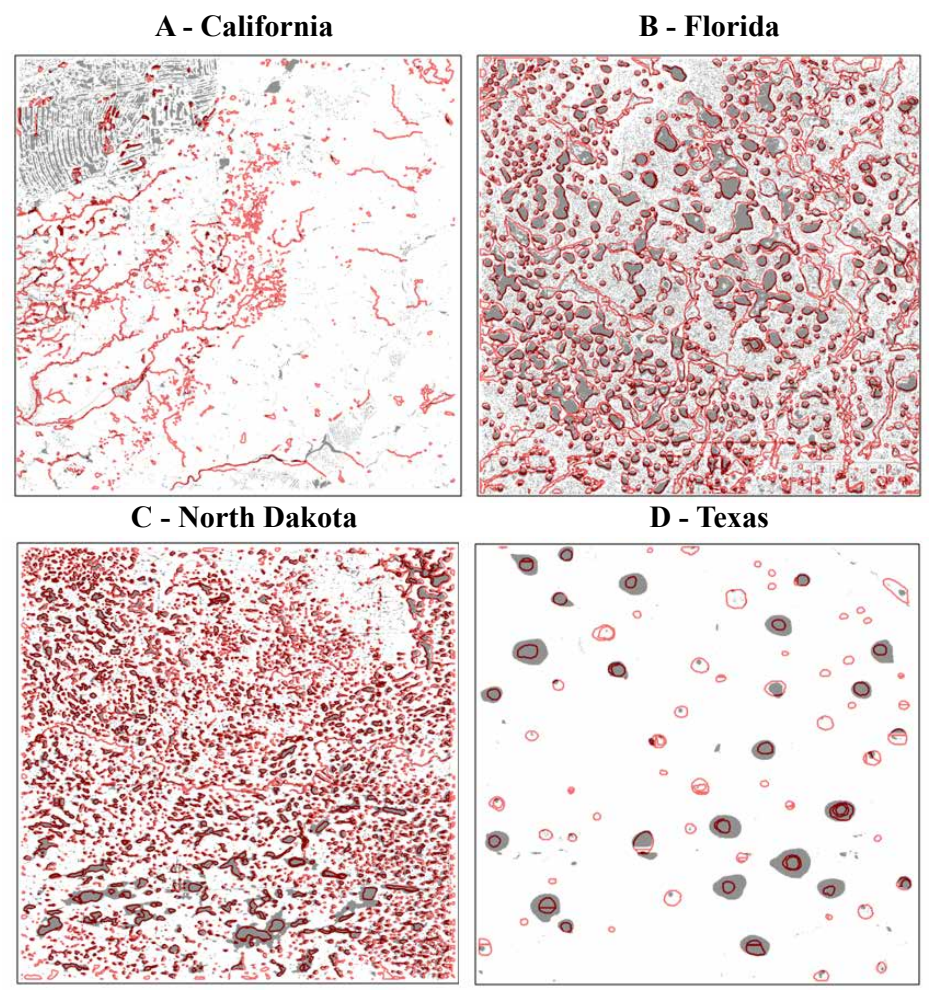

Figure 3: Comparison between the wetlands identified by the local search algorithm (grey regions) and the actual wetlands provided by the NWI database (red contours) for each wetlandscape. 